\title{
Effective diffusion of scalar fields in a chaotic flow
}

\author{
M. R. Turner, ${ }^{\text {a) }}$ A. D. Gilbert, and J. Thuburn \\ Mathematics Research Institute, School of Engineering, Computing and Mathematics, University of Exeter, \\ Exeter EX4 4QF, United Kingdom
}

(Received 7 May 2008; accepted 12 September 2008; published online 15 October 2008)

\begin{abstract}
The advection of a tracer field in a fluid flow can create complex scalar structures and increase the effect of weak diffusion by orders of magnitude. One tool to quantify this is to measure the flux of scalar across contour lines of constant scalar. This gives a diffusion equation in area coordinates with an effective diffusion that depends on the structure of the scalar field and, in particular, takes large values when scalar contours become very extended. The present paper studies the properties of this effective diffusion using a mixture of analytical and numerical tools. First the presence of hyperbolic stationary points, that is, saddles, in the scalar concentration field is investigated analytically, and it is shown that these give rise to singular spikes in the effective diffusion. This is confirmed in numerical simulations in which complex scalar fields are generated using a time-periodic flow. Issues of numerical resolution are discussed and results are given on the dependence of the effective diffusion on grid resolution and discretization in area or scalar values. These simulations show complex dependence of the effective diffusion on time as saddle points appear and disappear in the scalar field. It is found that time averaging (in the presence of an additional scalar source term) removes this dependence to leave robust results for the effective diffusion. () 2008 American Institute of Physics. [DOI: 10.1063/1.2998461]
\end{abstract}

\section{INTRODUCTION}

When a scalar tracer is injected into a fluid flow it is advected with the fluid and exposed to molecular diffusion, both of which form key aspects of the mixing properties of the flow. Advection stretches and folds the material lines of the scalar, and molecular diffusion removes the resulting fine scale structure in the scalar field. While advection and diffusion are important effects in scalar mixing processes, in general it is hard to separate their individual effects from one another. However, by defining a quasi-Lagrangian coordinate system based on the area inside scalar concentration contours the reversible effects of advection can be separated from the irreversible effects of diffusion. ${ }^{1,2}$ In this isotracer coordinate system the effects of advection are incorporated into the coordinate system and the advection-diffusion equation becomes a pure diffusion equation in area coordinates with an effective diffusion. This effective diffusion then quantifies solely the irreversible effects.

The effective diffusion from the quasi-Lagrangian approach measures the diffusive flux of the scalar tracer across concentration contours. It depends only on the instantaneous distribution of the scalar field and so can be calculated without knowledge of the flow field. However, when a fluid flow generates complex scalar fields, for example, by chaotic advection, the effective diffusion indirectly reveals information about the transport and mixing properties of the underlying flow. The simple nature of this approach and its ease of implementation make it an attractive tool for studying complicated systems such as the transport and mixing properties

\footnotetext{
${ }^{\text {a) }}$ Author to whom correspondence should be addressed. Electronic mail: m.r.turner@ex.ac.uk. Telephone: +441392-725280. Fax: +441392217965 .
}

of atmospheric flows. ${ }^{3,4}$ This diagnostic has been tested on a number of idealized flows to understand its properties. In a scalar field advected by the nonlinear Kelvin-Helmholtz instability, Nakamura ${ }^{1}$ found that regions of high effective diffusion occur in the center of a shear layer where the tracer (temperature in this case) is well mixed. Outside this well mixed region the effective diffusion drops off and this led Nakamura ${ }^{1}$ to conclude that there is a link between regions of high effective diffusion and well mixed regions of the tracer in the flow. Shuckburgh and Haynes ${ }^{5}$ made a careful study of effective diffusion as a rigorous diagnostic for quantifying flows and they strengthened the link between regions of high effective diffusion and well mixed regions showing that high effective diffusion occurs in regions of high material line stretching. This was achieved by studying a simple two-dimensional time-periodic flow with the form of a meandering jet and comparing the effective diffusion with other Lagrangian mixing diagnostics such as Poincaré sections ${ }^{6,7}$ and finite-time Lyapunov exponents. ${ }^{8,9}$ In fact it was found that the effective diffusion was better able to distinguish barriers in the flow when compared with finite-time Lyapunov exponents. $^{5}$

As the effective diffusion is easy to implement and no prior knowledge of the velocity field is required, this method is appealing to experimentalists too. The scalar concentrations can be calculated using grayscale images and thus the required integrals in the effective diffusion (see Sec. II) can be calculated by counting pixels, e.g., see Deese $e t$ al. ${ }^{10}$

The purpose of this paper is also to examine the robustness of effective diffusion as a diagnostic using twodimensional scalar fields given analytically or from advection-diffusion in idealized flows. Our starting point is to understand singular behavior in the effective diffusion, 
which arises when the scalar field contains stationary points that are saddles, that is, have locally hyperbolic contour lines. This could be significant because in the evolution of a complex field such saddles will be continually created and destroyed, leading to complex behavior of the effective diffusion and opens the question of how useful a diagnostic it really is.

We begin by considering an instantaneous snapshot of a saddle point for which we can write down the scalar field analytically. Using numerical methods we show that the effective diffusion along the concentration contour through the saddle point is singular, and we confirm analytically that the singularity is logarithmic. We then study scalar advected by an incompressible time-periodic flow, with a fixed steady source of scalar so as to produce a strictly time-periodic scalar distribution. We observe that over a period of the flow the saddle points of the scalar field move through the region of high effective diffusion, corresponding to the well mixed region of the flow. We also determine the numerical resolution required to obtain robust results and how time averaging may be used to obtain a mean eddy diffusion without complex time dependence and singularities that then characterizes the mixing over one period of the underlying flow. Our motivation for this study is to test the robustness of the effective diffusion diagnostic so that it may be used on dynamical vortex problems where the vorticity field may contain saddle points in highly nonlinear regimes such as during the formation of cat's eyes. ${ }^{11}$

The paper is set out as follows. In Sec. II we state the form of the transformed advection-diffusion equation, define the effective diffusion, and give the numerical method, following previous work. In Sec. III we use a two-dimensional analytical scalar field to show that the effective diffusion has a logarithmic singularity at tracer values where contours pass through a saddle point. This information is then used in the numerical simulations in Sec. III C to show that peaks in the effective diffusion relate directly to saddle points in the scalar field. In Sec. III D we introduce a source of scalar into the simulations so as to produce a time-periodic scalar field. By time averaging, we demonstrate that high values of the timeaveraged effective diffusion correspond to regions in the scalar field which are well mixed and where the material lines are highly stretched. Our concluding remarks and comments are given in Sec. IV.

\section{FORMULATION}

In an incompressible fluid, the evolution of a scalar tracer with concentration $c(\mathbf{x}, t)$ is given by the advectiondiffusion equation

$$
\frac{\partial c}{\partial t}+\mathbf{u} \cdot \nabla c=\nabla \cdot(\kappa \nabla c)+S,
$$

where $\kappa(\mathbf{x}, t)$ is the molecular scalar diffusion, $S$ is a source term, and $\mathbf{u}$ is a prescribed incompressible velocity field. By making a transformation to isotracer coordinates, ${ }^{12}$ Eq. (1) reduces to a diffusion-only equation for the time evolution of the scalar as a function of area in two dimensions ${ }^{1}$ or volume in three dimensions. ${ }^{2}$ We define the area or volume of a scalar contour $C$ as the region bounded by this contour or surface, i.e., everywhere where the scalar concentration $c(\mathbf{x}, t)$ is less than or equal to $C$. Therefore we write

$A=\iint H(C-c(\mathbf{x}, t)) d A, \quad V=\iiint H(C-c(\mathbf{x}, t)) d V$,

where $H(X)$ is the Heaviside function. Thus in two dimensions, $A=0$ when $C=C_{\min }$ and $A=A_{\max }$ when $C=C_{\max }$ using obvious notation. We also define $\gamma(C, t)$ to be the bounding contour or surface of the region where $A$ or $V$ is less than $C$ at time $t$.

Under this change of coordinates the two-dimensional scalar evolution equation becomes

$$
\frac{\partial C(A, t)}{\partial t}=\frac{\partial}{\partial A}\left(K_{\mathrm{eff}}(A, t) \frac{\partial C(A, t)}{\partial A}\right)+\langle S\rangle_{A},
$$

with an effective diffusion that can be written as

$$
K_{\text {eff }}=\frac{\partial A}{\partial C} \int_{\gamma(C, t)} \kappa|\nabla c| d s
$$

or as

$$
K_{\mathrm{eff}}=\frac{\left\langle\kappa|\nabla c|^{2}\right\rangle_{A}}{(\partial C / \partial A)^{2}},
$$

where the weighted contour average of a quantity $\chi$ is given by

$$
\langle\chi\rangle_{A}=\frac{\partial}{\partial A} \int_{c \leq C(A, t)} \chi d A=\left(\int_{\gamma} \frac{d s}{|\nabla c|}\right)^{-1} \int_{\gamma} \chi \frac{d s}{|\nabla c|}
$$

(see Refs. 1 and 5). A similar equation holds in three dimensions, with $A$ replaced by $V$. Throughout this work we assume that the molecular diffusion $\kappa(\mathbf{x}, t)$ is constant; hence $\kappa$ can be taken outside the integrals in Eqs. (4) and (5).

\section{A. Numerical method for calculating the effective diffusion}

When we consider a two-dimensional scalar field that is defined analytically, the effective diffusion may be calculated using Eq. (4). This can be manipulated and evaluated simply as a single integral over the $x$ coordinate (see Sec. III A for more details). However, when we consider flows where the scalar concentration is only known from a full numerical simulation of Eq. (1), we use Eq. (5) to calculate $K_{\text {eff }}$ at a given time value using the following algorithm. ${ }^{5}$ First we define a set of $C$ values from $C_{\min }$ to $C_{\max }$ with a constant spacing $\Delta C$, and for each of these values $A(C)$ is calculated via Eq. (2). The function $A(C)$ is calculated using a box counting technique, where we assume that the value of the scalar at the grid point gives the scalar value in the entire grid box surrounding that point. Then the square of the scalar gradient, $|\nabla c|^{2}$, is calculated at each grid point, and this quantity is integrated over the area bounded by the desired tracer contour to obtain $\int_{c \leq C(A, t)}|\nabla c|^{2} d A$ using the same box counting. Taking central finite differences of this integral with respect to the area coordinate then gives $\left\langle|\nabla c|^{2}\right\rangle_{A}$, which 


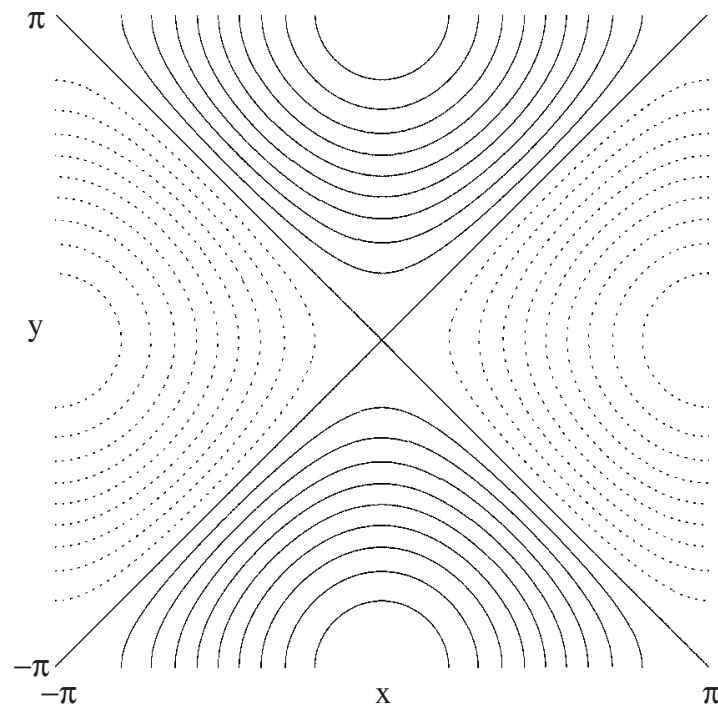

FIG. 1. Contour plot of scalar field (7) showing a saddle point at the origin. Negative concentration contours are dashed and positive contours are solid.

on dividing by $(\partial C / \partial A)^{2}$ and multiplying by $\kappa$ gives $K_{\text {eff }}$. Like the term $\left\langle|\nabla c|^{2}\right\rangle_{A}$, the quantity $\partial C / \partial A$ is calculated using a central difference scheme, and so $K_{\text {eff }}$ is prone to numerical error arising from these terms, as is discussed in more detail in Sec. III B. The effective diffusion in three dimensions is calculated in a similar fashion, with volume integrals replacing the area integrals. The accuracy of $K_{\text {eff }}$ in conjunction with the resolution of numerical simulations is discussed in Sec. III B.

\section{EFFECTIVE DIFFUSION IN TWO DIMENSIONS}

\section{A. Effective diffusion in scalar fields with saddle points}

Consider the scalar field in two dimensions given by

$$
c(x, y)=\frac{1}{2}(\cos x-\cos y)
$$

in the periodic, Cartesian domain $[x, y] \in[-\pi, \pi)$. A contour plot of this scalar field is given in Fig. 1, where the dashed contours are negative and the solid ones are positive. This scalar field has two saddle points, one at $(0,0)$ and the other at $(-\pi,-\pi)$, both of which lie on the scalar concentration contour $C=0$. (These saddle points are the two distinct saddle points; the others present are due to the periodicity of the scalar field.) The area $A$ inside the scalar concentration contour $C$ can thus be calculated from Eq. (2) as

$$
A=\int_{-\pi}^{\pi} \int_{-\pi}^{\pi} H(2 C-\cos x+\cos y) d x d y .
$$

This quantity is evaluated numerically, and $\partial A / \partial C$ is calculated via finite differences. The function $A(C)$ for this scalar field can be seen as the solid line in Fig. 2(a). Because of the symmetry of the scalar field, $A(C)$ is an odd function with a rapid increase in area around $C=0$. We shall see later that the gradient at $C=0$ is in fact infinite and it is this infinite gradient that leads to a singularity in $K_{\text {eff }}$.

To calculate the effective diffusion we need the quantity

$$
F(C)=\int_{\gamma(C)}|\nabla c| d s
$$

from Eq. (4), which (apart from a factor $\kappa$ ) is minus the diffusive flux of scalar over the contour $\gamma(C)$. This flux can be transformed for $C>0$ to

$$
F(C)=8 \int_{0}^{\rho} \frac{|\nabla c|^{2}}{|\sin y|} d x
$$

where $\rho=\cos ^{-1}(2 C-1)$. Here we have used the fact that

$$
\frac{d s}{d x}=\left(1+\frac{\sin ^{2} x}{\sin ^{2} y}\right)^{1 / 2}=\frac{2|\nabla c|}{|\sin y|}
$$

and that the contour $C$ hits the line $y=\pi$ at $x=\rho$ in the upper right quadrant. The same expression holds for $C<0$ by symmetry. Using Eq. (7) we can write $F(C)$ solely in terms of $x$ by noting that

$|\sin y|=\left(1-\cos ^{2} y\right)^{1 / 2}=\left(1-\cos ^{2} x+4 C \cos x-4 C^{2}\right)^{1 / 2}$.

A numerical integration for $F(C)$ and so $K_{\text {eff }}(C)$ for this scalar distribution are shown as the solid lines in Figs. 2(b) and 2(c), respectively, where we have set $\kappa=1$. The plot of $K_{\text {eff }}(C)$ appears to have a singularity at $C=0$, and because $F(C)$ in Fig. 2(b) appears to be well behaved at $C=0$, then the singularity must arise from the term $\partial A / \partial C$.

To check the nature of this singularity we expand Eqs. (8) and (10) for small $C$ to show that $K_{\text {eff }}$ has a logarithmic singularity along the concentration contour $C=0$. The calculations are included in Appendix A, and we just quote the results:

$$
\begin{aligned}
& A(C)=-8 C \ln |C|+2 \pi^{2}+19.07 C+O\left(C^{2}\right), \\
& F(C)=4 C^{2} \ln |C|+8-15.1 C^{2}+O\left(C^{3}\right), \\
& K_{\mathrm{eff}}(C)=-64 \ln |C|+88.56+O(C \ln |C|) .
\end{aligned}
$$

From these expansions we see that in fact $F(C)$ is not well behaved at $C=0$, but its singularity is weaker than the one from $\partial A / \partial C$ which is $O(\ln |C|)$. These asymptotic expansions are plotted as the dashed lines with their numerical equivalents in Fig. 2. We see that the asymptotic results and the numerical results are in excellent agreement around $C=0$, and in fact the asymptotic results for $A(C)$ are graphically indistinguishable from the numerical results for $-0.5<C<0.5$.

This logarithmic singularity in $K_{\text {eff }}$ is not just restricted to the case where two saddle points have the same concentration contour passing through them. As a further example we consider the scalar field

$$
c(x, y)=\frac{1}{2}(\cos x-\alpha \cos y),
$$

with $0<\alpha<1$. A contour plot of this scalar field with $\alpha=0.5$ is shown in Fig. 3. We examine the numerical integration for $K_{\text {eff }}(A)$ with $\alpha=1.0,0.75,0.5$, and 0.25, shown in Fig. 4. As $\alpha$ is reduced from 1 in Fig. 4, the single singular point at $A=2 \pi^{2}(C=0)$ becomes two singular points with a region of lower effective diffusion in between. For $\alpha<1$ the saddle point at $(0,0)$ now has the scalar concentration value 

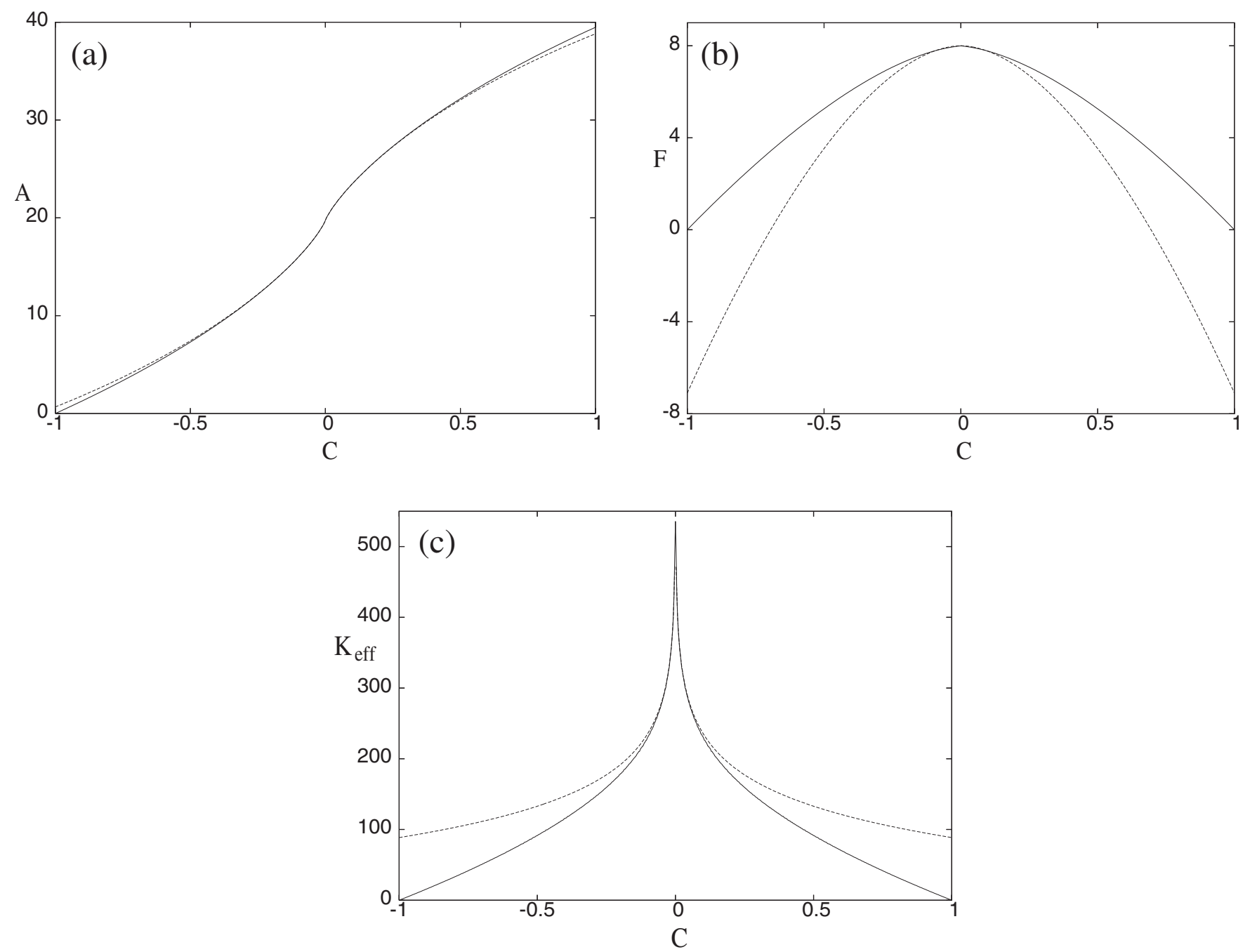

FIG. 2. Plots of (a) $A(C)$, (b) $F(C)$, and (c) $K_{\text {eff }}(C)$ with the numerical results plotted as the solid lines and the small $C$ asymptotic results as the dashed lines for scalar field (7).

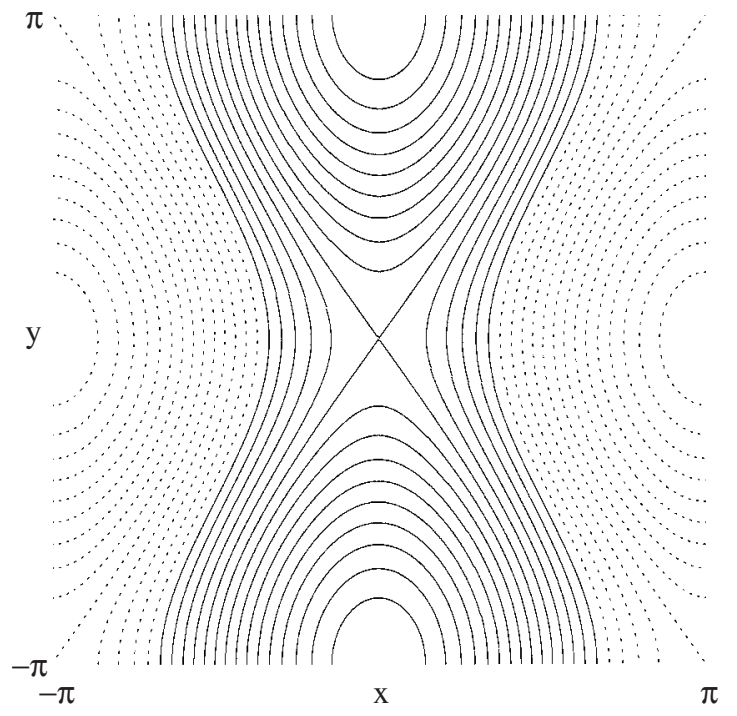

FIG. 3. Contour plot of scalar distribution (14) with $\alpha=0.5$ showing two saddle points, one at the origin and one at $(-\pi,-\pi)$. Negative contours are dashed and positive contours are solid.

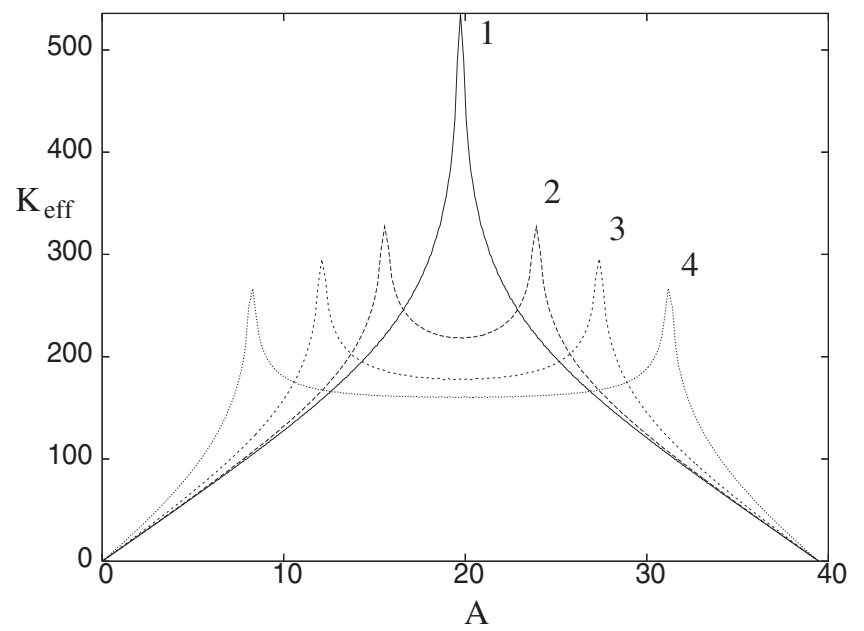

FIG. 4. Plot of $K_{\text {eff }}(A)$ for scalar distribution (14) with $\kappa=1$ and $\alpha=1,0.75$, 0.5 , and 0.25 corresponding to lines $1-4$, respectively. 

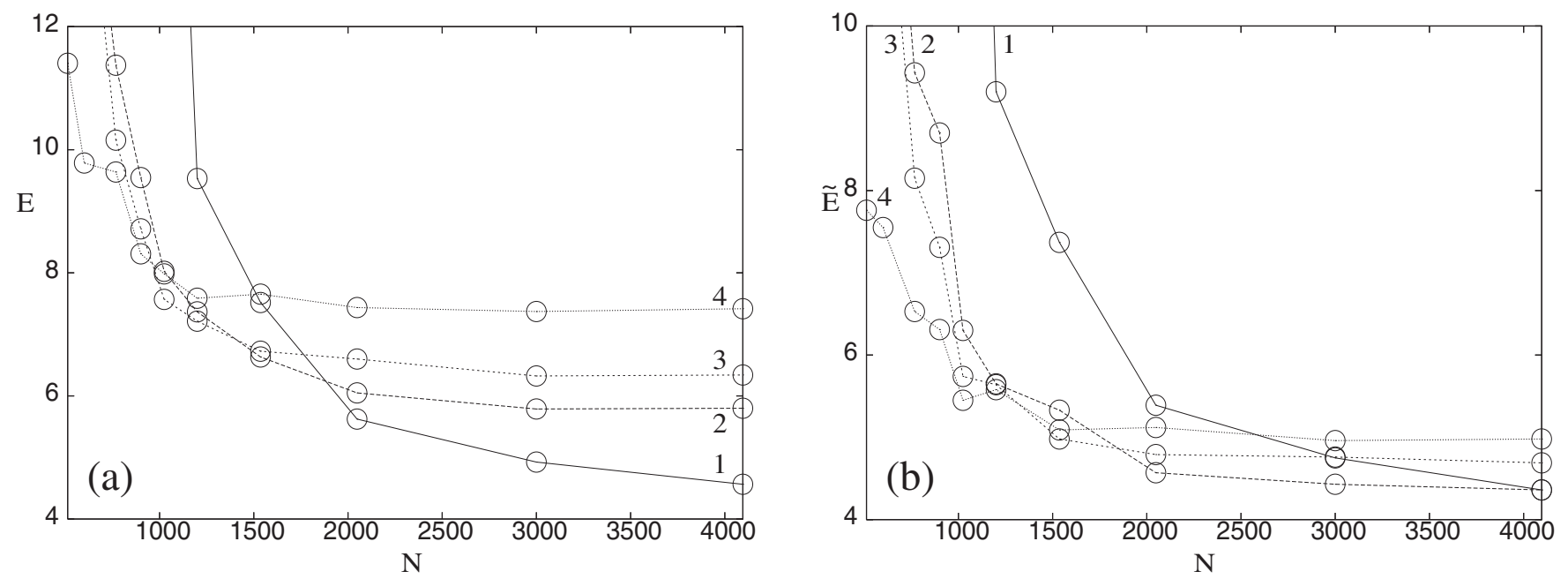

FIG. 5. Plot of (a) the mean square error $E$ and (b) the capped mean square error $\widetilde{E}$ as a function of the size of the simulation $N$ for $\Delta C=0.0025 C_{\max }$ (line 1), $0.005 C_{\max }$ (line 2), $0.0075 C_{\max }$ (line 3), and $0.01 C_{\max }$ (line 4). In panel (b) the calculation does not include points where $K_{\text {eff }}^{\text {true }} \geq 250$.

$C=(1-\alpha) / 2>0$, which is different from the saddle point at $(-\pi,-\pi)$, which has the value $C=-(1-\alpha) / 2<0$. For the case $\alpha=0.5$ these correspond to $C= \pm 1 / 4$, respectively, which themselves correspond to the values of $A$ given in Fig. 4. The reason we plot these results as a function of $A$ rather than $C$ is because varying $\alpha$ changes the upper and lower limits of $C$, while the upper and lower limits of $A$ remain fixed at $A_{\max }=4 \pi^{2}$ and $A_{\min }=0$, respectively. For this reason, in many figures in this paper we will use $A$ as the independent variable, so as to obtain the same range no matter what the concentration field limits are. As $\alpha$ is reduced toward zero the singularities move toward the limiting area values $A_{\min }=0$ and $A_{\max }=4 \pi^{2}$, and the region of almost constant diffusion between the singular points extends until for $\alpha=0$ the diffusion will be constant everywhere and $c(x, y)=\frac{1}{2} \cos x$.

We note that the logarithmic singularity in $K_{\text {eff }}$ in two dimensions is not present in the equivalent three-dimensional calculation, which is given in Appendix B.

\section{B. Parameter optimization for two-dimensional numerical simulations}

When we consider an analytically defined scalar field as in Sec. III A, we are able to obtain $K_{\text {eff }}$ with a high degree of accuracy by increasing the resolution of the integrals in Eqs. (2) and (4). However, in numerical simulations our accuracy is restricted by the resolution of the simulation, so in this section we assess the optimal parameters for the numerical evaluation of $K_{\text {eff }}$ for a scalar field generated under advection-diffusion with a given molecular diffusion $\kappa$.

When calculating the effective diffusion using the numerical method outlined in Sec. II A, there are two parameters that we can vary. These are the concentration step size $\Delta C$ and $N$, which is the resolution of our simulation computed on an $N \times N$ computational grid. (Note that the true level of structure in the scalar field depends on $\kappa$ and that decreasing $\kappa$ just requires reducing $\Delta C$ to pick out the fine structure of $K_{\text {eff }}$, and so variations in $\kappa$ can be considered as variations in $\Delta C$ for optimizing purposes.) Unfortunately the parameters $\Delta C$ and $N$ are not independent because for a given simulation, reducing $\Delta C$ requires an increase in $N$ to retain the accuracy of the results: a smaller $\Delta C$ introduces numerical oscillations in $K_{\text {eff }}$ through the finite difference method. A larger value of $N$ will overcome this by producing more accurate evaluations of the integral in Eq. (4) before the finite difference procedure is applied. An increase in $\Delta C$ on the other hand would allow for a smaller value of $N$; however, then some fine scale structure in $K_{\text {eff }}$ may be missed. Thus for any value of $N$ there is an optimal value of $\Delta C$ which minimizes the numerical oscillations but also maximizes the physical features that are resolved in $K_{\text {eff }}$. To study this issue we use the numerical scheme defined in Sec. II A to calculate the effective diffusion for a scalar field which is known analytically so that we also know $K_{\text {eff }}$ to a high degree of accuracy using form (4). For this optimization study we use the scalar field given by Eq. (14) with $\alpha=0.5$, and we call the highly accurate solution the "true solution."

To calculate the error between the effective diffusion calculated using the method in Sec. II A and the true solution we use a mean square error given by

$$
E=\frac{1}{M} \sum_{i=1}^{M}\left[K_{\mathrm{eff}}(i)-K_{\mathrm{eff}}^{\mathrm{true}}(i)\right]^{2},
$$

where $K_{\text {eff }}^{\text {true }}$ is the true form of the effective diffusion and $K_{\text {eff }}(i)$ is the effective diffusion calculated at the $M$ values of $C$. This error is plotted in Fig. 5(a) as a function of the simulation size $N$ for $\Delta C=(0.0025,0.005,0.0075,0.01) C_{\max }$ represented by lines $1-4$, respectively. We choose $\Delta C$ to be a multiple of $C_{\max }$ because then regardless of the value of $C_{\text {max }}, K_{\text {eff }}$ will still have the same number of points distributed along the $A$ axis, which is the quantity against which we plot $K_{\text {eff }}$. This is a reasonable choice of $\Delta C$ for the simulations discussed in this paper.

For small values of $N$ we see in Fig. 5(a) that small $\Delta C$ values give a poor agreement with the true solution because of the numerical error from the finite difference methods for both the numerator and denominator of $K_{\text {eff }}$, introducing 
large oscillations into $K_{\text {eff. }}$ As $N$ is increased, the smallest value of $\Delta C$ eventually gives the smallest error at around $N=2000$. However, when we examine a plot of $K_{\text {eff }}$ for this value of $N$ and $\Delta C$ (not shown here) we find that the actual agreement with the true solution is good around the peaks in $K_{\text {eff }}$, but over the rest of the function there still exist large numerical oscillations, which makes the agreement quite poor. The reason for this is that the mean square error is dominated by the singular peaks in $K_{\text {eff }}$, so well resolved peaks can mean a small error even if the agreement over the rest of the function is visually poor. Thus we remove the peaks from the error calculation by modifying the mean square error in Eq. (15) to exclude any points where $K_{\text {eff }}^{\text {true }} \geq 250$, and we call this $\widetilde{E}$. A plot of $\widetilde{E}$ as a function of $N$ is plotted in Fig. 5(b) with the same values of $\Delta C$ as in panel (a). Comparing the results in this panel to those in panel (a) we see that where the $\Delta C=0.0025 C_{\max }$ result gave the smallest error for $N>2000$, we see that this value now does not give the smallest error until $N \approx 4096$. This shows that although the small $C$ increments allow for a better approximation of the peaks in $K_{\text {eff }}$ it does not approximate the rest of the function as well as $\Delta C=0.005 C_{\max }$ does.

For the numerical simulations in this paper we shall use $N=2048$ because this is large enough to give a fine grid resolution but small enough so that it is not computationally expensive. Hence guided by the results in Fig. 5(b) we use an increment in $C$ of $\Delta C=0.005 C_{\max }$ to produce the best numerical results. Although we are not able to give a general optimized step size $\Delta C(N)$ for all problems, for periodic flows like those considered in this paper, a reasonable concentration step size would be $\Delta C \approx 10 C_{\max } / N$.

\section{Two-dimensional numerical simulations with no source term}

We now consider scalar fields generated numerically by advection and diffusion: specifically we consider a scalar concentration $c(x, y, t)$ whose evolution is governed by Eq. (1) with $\kappa$ constant and with no source term $(S=0)$. The fluid velocity is taken to have the unsteady form

$$
\mathbf{u}=2 \cos ^{2} t(0, \sin x)+2 \sin ^{2} t(\sin y, 0),
$$

and initially $c(x, y, 0)=2 \cos x$. The numerical scheme for solving Eq. (1) transforms the $N \times N$ domain into Fourier space by writing

$$
c(x, y, t)=\sum_{j=-N}^{N} \sum_{k=-N}^{N} \hat{c}_{j k}(t) e^{-i(j x+k y)},
$$

where the time integration of the advection is performed via the second order Adams-Bashforth method and diffusion is integrated exactly. It is well known that this velocity field generates island regions of recirculation, separated by bands of chaotic mixing. ${ }^{13,14}$ These features are reflected in the evolution of the advected scalar field, for example, the "whorls" and "tendrils" of Berry and Balazs ${ }^{15}$ or the mirroring of the saddle points and unstable manifold structure of the flow in the advected scalar contours of Ghosh et al. ${ }^{16}$ Thus, with mild abuse of language, we refer to islands and
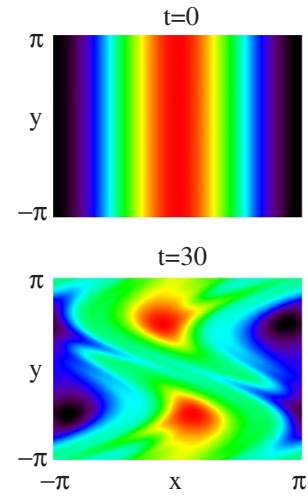

$t=10$

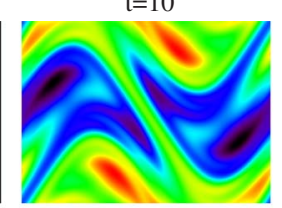

$\mathrm{t}=40$

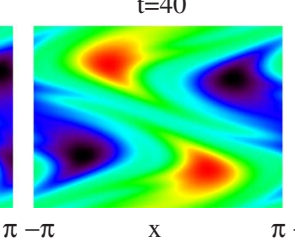

$\mathrm{t}=20$

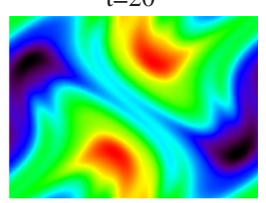

$\mathrm{t}=50$

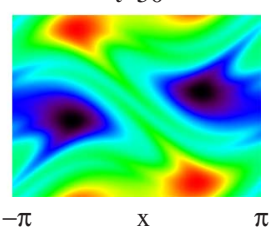

FIG. 6. (Color online) Plot of the scalar field $c(x, y, t)$ for the numerical simulations at $t=0,10,20,30,40$, and 50 , with $\kappa=0.01$. In each figure the maximum scalar value at each time is colored medium gray (red online) and the lowest value is black.

chaotic regions of the scalar field, corresponding to these regions of the flow.

With the initial condition $c(x, y, 0)=2 \cos x$, the simulations can be thought of as starting with a scalar field with constant effective diffusion and allowing it to evolve toward a distribution similar to that in Eq. (7) except with some time dependence included. Panels showing the scalar distribution for $\kappa=0.01$ at times $t=0,10,20,30,40$, and 50 are shown in Fig. 6, where for $t \geq 30$ we can distinguish the two pairs of islands (seen colored medium gray or red online at the top and bottom of the panels and black or dark blue online on the left and right of the panels) which trap scalar, and the region of rapid chaotic mixing in between (see also Fig. 10 below). For $t=10$ the scalar field has a rather disorganized structure when compared to the $t=50$ panel, with no single region of coherent high and low scalar concentrations. However, as time increases to $t=50$ the scalar becomes more organized with two coherent regions of negative scalar at the left and right of the panel and two positive regions of scalar at the top and bottom of the panel. Thus we might expect the scalar for $t=10$ to contain more saddle points and $K_{\text {eff }}$ to be more complicated when compared to $K_{\text {eff }}$ for the $t=50$ distribution. We also expect $K_{\text {eff }}$ for $t=50$ to have its largest values in the chaotic mixing region where the filament lines are most stretched.

Figure 7 plots the effective diffusion $K_{\text {eff }}(A)$ at $t=10$ for the above simulation with fixed $\Delta C=0.005 C_{\max }$ and $N=512,1024,2048$, and 4096. The plots are separated by an additive constant with the $N=512$ result at the bottom and the $N=4096$ result at the top of the panel. The lowest resolution simulation, $N=512$, produces a $K_{\text {eff }}$ with fine scale oscillations and many sharp peaks that could be mistaken as features of $K_{\text {eff. }}$. These oscillations are clearly visible between $A=16$ and 23. However, as $N$ is increased many of these large oscillations decrease or disappear, showing that they are merely numerical error from the finite difference calculations of the numerator and denominator of $K_{\text {eff. }}$ For the large $N$ simulations there are a few clear peaks left in $K_{\text {eff, }}$, which can be shown to correspond to saddle points in the scalar field distribution, see Fig. 9 below.

Figure 8 shows the effective diffusion $K_{\text {eff }}(A)$ for the 


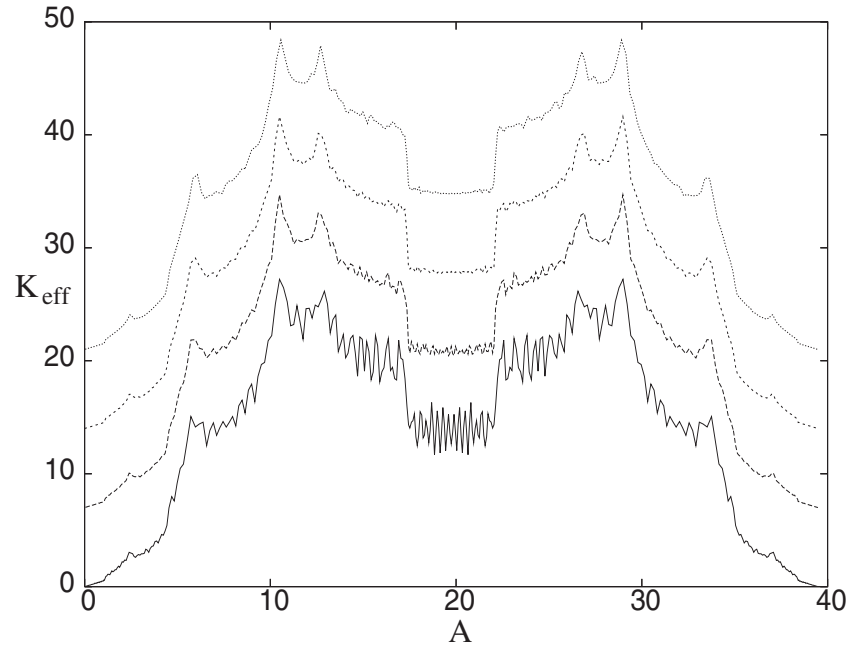

FIG. 7. Plot of $K_{\text {eff }}(A)$ for $t=10$ from Fig. 6 with $N=512,1024$, 2048, and 4096 and $\Delta C=0.005 C_{\max }$. The results are separated by an additive constant, with $N=512$ at the bottom and $N=4096$ at the top.

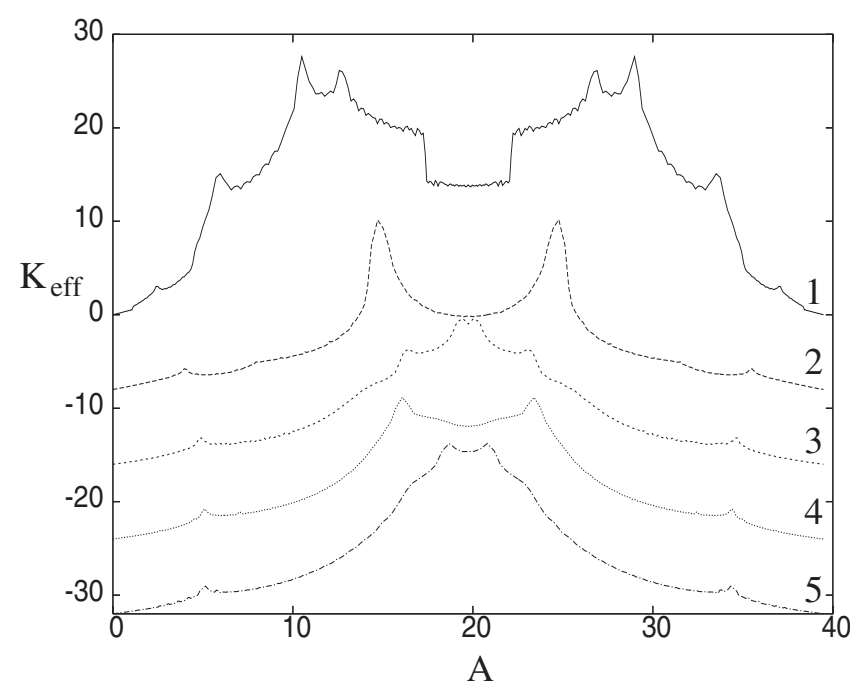

FIG. 8. Plot of $K_{\text {eff }}(A)$ for the panels shown in Fig. 6. Lines 1-5 represent times $t=10$ to $t=50$, respectively, and are separated by a constant for clarity. simulations in Fig. 6 at the times $t=10,20,30,40$, and 50, numbered $1-5$, respectively. These results are separated by a constant for clarity. We see that at $t=10$ (line 1) the effective diffusion has many small peaks and troughs, as discussed in the previous paragraph. This behavior could be anticipated from the $t=10$ panel in Fig. 6, which shows a very complicated distribution containing saddle points corresponding to the peaks in $K_{\text {eff. }}$ At $t=20$ (line 2) the scalar field appears more organized than that at $t=10$. This is reflected in $K_{\text {eff }}$ where we now only see four clear peaks close to $A=4,15$, 25 , and 36, respectively. As time increases (see lines 3-5) the two peaks close to $A=2 \pi^{2}$ move together and combine into one large peak. The other two peaks move closer to $A=5$ and $A=35$ and persist for long times, although the effective diffusion at these points is small compared to the large diffusion across contours close to $A=2 \pi^{2}$.

Figure 9(a) plots $K_{\text {eff }}$ as a function of $C$ at $t=20$; this is a stretched version of line 2 from Fig. 8, where $K_{\text {eff }}$ is depicted as a function of $A$. We can now read off that the peaks occur at $C \approx \pm 0.08$ and $C \approx \pm 0.35$. Thus in Fig. 9(b) we plot the $t=20$ panel from Fig. 6 as a contour plot, with the five contour levels $-0.07,-0.079,-0.09,-0.345$, and -0.355 . Examining these contours we can clearly see two saddle points situated at the points marked with an $X$. These points correspond to the peaks in panel (a) at $C \approx-0.08$ and -0.35 , thus confirming that peaks in the effective diffusion in two dimensions are due to logarithmic singularities which occur at concentration contours passing through saddle points.

\section{Two-dimensional numerical simulations with a source term}

We have seen that the effective diffusion shows quite complex time dependence as saddle points are created, destroyed, and moved within the scalar field. The obvious solution is to perform a time average. However, the scalar distribution decays with time and so the system is not in a stationary state. Furthermore if the system is run for a long time, the scalar field will become constant (at least to nu-
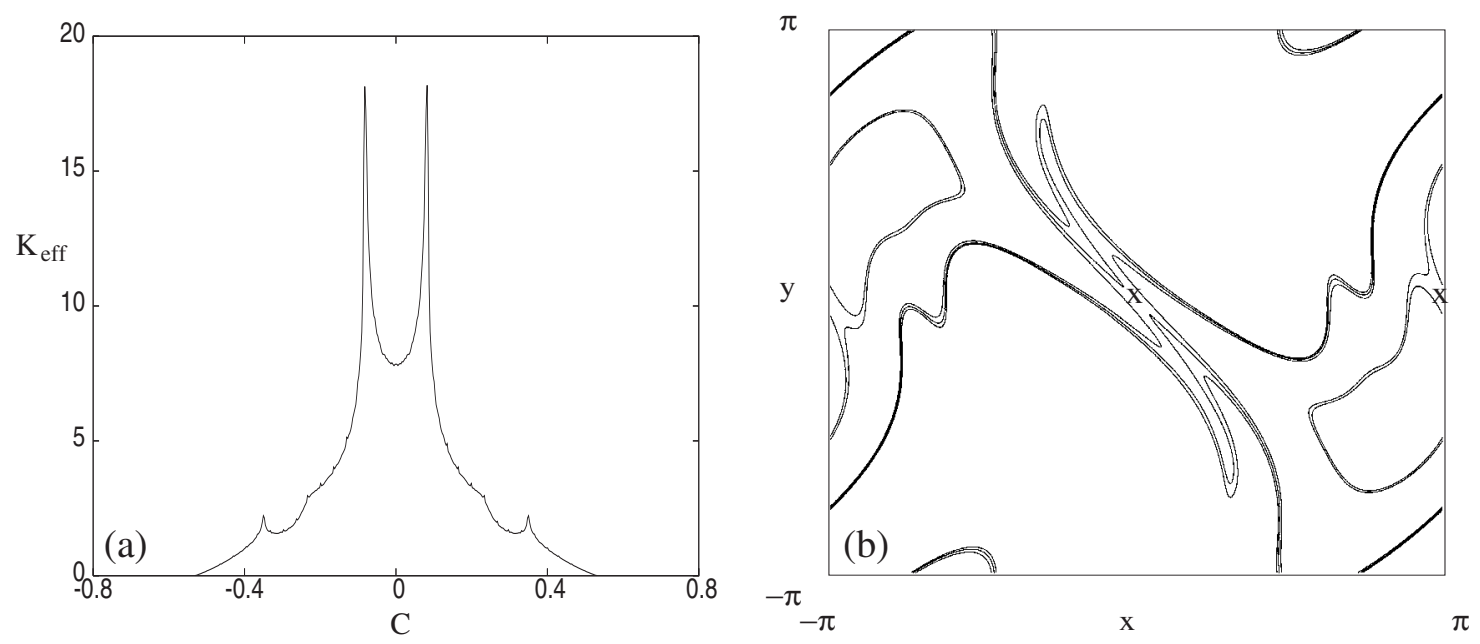

FIG. 9. Plot of (a) $K_{\text {eff }}(C)$ for $\kappa=0.01$ at $t=20$ and (b) the corresponding contour plot from Fig. 6 with contour levels given at $-0.07,-0.079,-0.09,-0.345$, and -0.355 . The two saddle points corresponding to the singular values of $C$ in panel (a) are represented by $X$ in panel (b). 
merical accuracy) in well mixed regions while slowly decaying in any island that is present. These potential problems were indicated by Shuckburgh and Haynes, ${ }^{5}$ who suggested introducing a source term to give a stationary state with nontrivial scalar structure in the well mixed regions.

In this section we therefore introduce a source term $S=\cos x$ into Eq. (1) and initially set $c(x, y, 0)=0$. This guarantees that a strictly periodic scalar field will be established in the asymptotic limit $t \rightarrow \infty$. However, for practical purposes this is still not entirely satisfactory: we find that the effective diffusion rapidly approaches an approximately periodic state in the chaotic band region. However, scalar slowly builds up in the islands (i.e., $C_{\max }$ slowly increases with time), where advection and diffusion operate on long time scales (of the order of $\kappa^{-1}$ ) and this scalar slowly leaks into the chaotic region. Although it must eventually saturate, the time scale for this is beyond that of our simulations. On the other hand even if there is a slow evolution of the island concentrations over a long time scale, we may anticipate that the effective diffusion will equilibrate quickly since the effective diffusivity is invariant under rescaling of the scalar field. In any case we take a pragmatic approach and use the scalar fields with moderate $\kappa$ obtained on these time scales to study the evolution of the scalar field over one period of the flow $(T, T+\pi)$. We then time average $K_{\text {eff }}$ and investigate how this quantity varies with both the period start time $T$ and $\kappa$

The approximately periodic state that is established in the chaotic region for scalar (16) can be seen in Fig. 10 which shows $|c(x, y, t)|_{c \leq 5}$ (left panels) and the corresponding $K_{\text {eff }}(A)$ (right panels) for $t=10 \pi$ to $t=11 \pi$ in increments of $\pi / 4$ with $\kappa=0.01$. In the real space panels the scalar field is capped at $|c|=5$ to eliminate islands and so emphasize the chaotic region of the flow where large effective diffusion occurs and the line filaments are stretched the most. The center of the chaotic band (medium gray region, red region online) is close to $|c|=0$ and the black regions are regions where $|c|>5$.

By considering the right-hand panels in Fig. 10 we see that over one period of the flow, the effective diffusion varies a considerable amount for $10<A<30$ but outside of this range the variation is small. At $t=10 \pi$ [Fig. 10(a)] $K_{\text {eff }}$ has two spikes of large effective diffusion around $A=2 \pi^{2}$ and two other peaks around $A=12$ and 28. Considering only $A>2 \pi^{2}$, the peak in $K_{\text {eff }}$ closest to $A=2 \pi^{2}$ occurs at approximately $(\pi / 2,0)$ in the real space plot, while the other peak in $K_{\text {eff }}$ corresponds to a saddle point at the origin. At $t=10 \pi+\pi / 4$ [Fig. 10(b)] the saddle point near $(\pi / 2,0)$ has practically been diffused away and this can be seen by the increase in the medium gray colored region (center of the chaotic band, red online) in this area. The other saddle point on the other hand begins to intensify and the effective diffusion peaks grow until at $t=10 \pi+3 \pi / 4$ [Fig. 10(d)] it is close to its maximum value. Beyond this time, $t=11 \pi$ [Fig. 10(e)], the flow returns the scalar approximately to its $t=10 \pi$ distribution.

The movement of the scalar in time is related to the flux of the scalar which itself is related to the rate of change in the concentration contours via
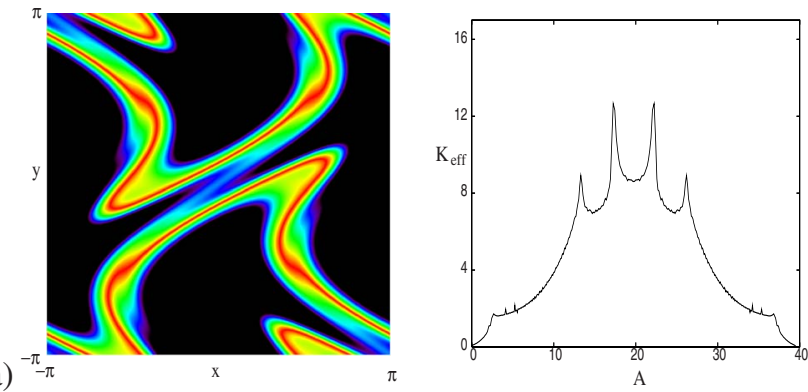

(b)
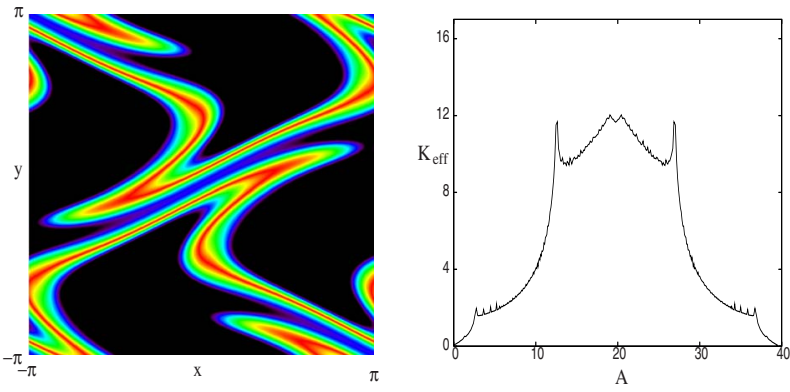

(c)
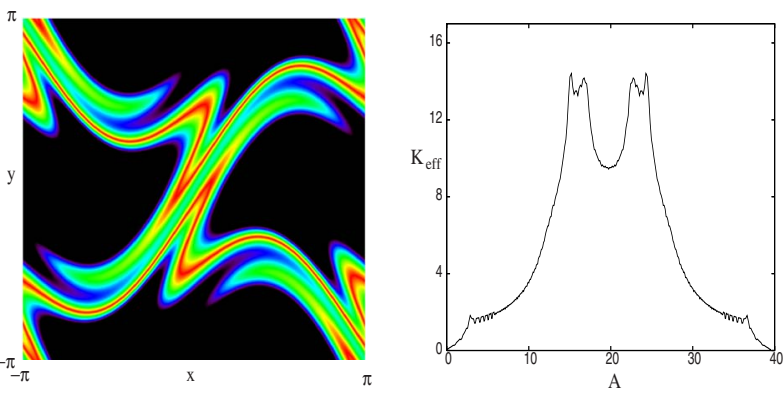

(d)
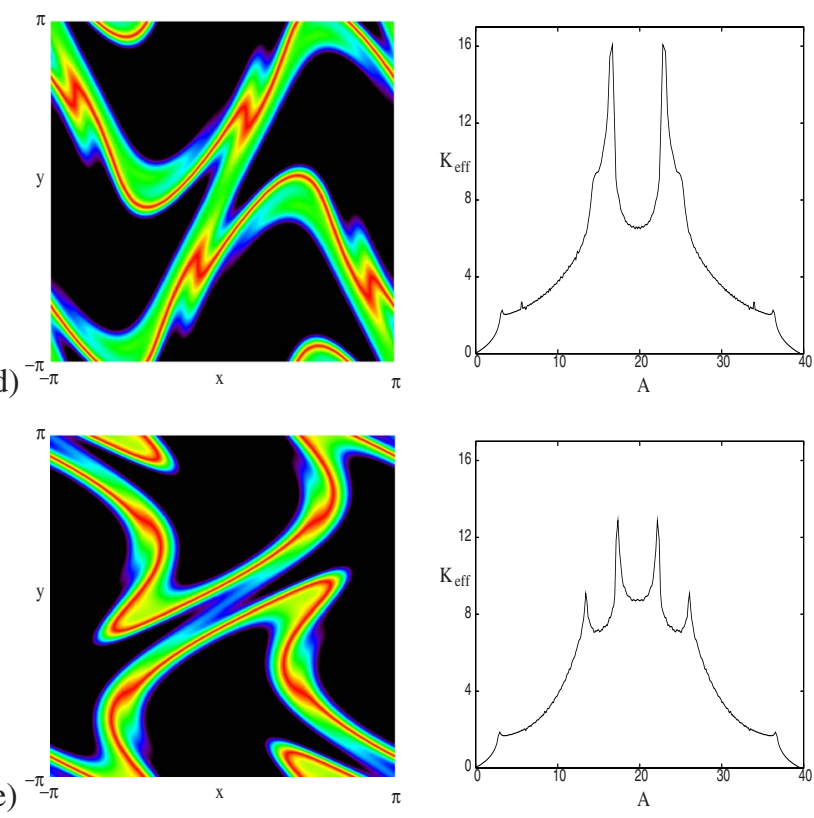

FIG. 10. (Color online) Plot of $|c(x, y, t)|_{c \leq 5}$ and the corresponding effective diffusion $K_{\text {eff }}(A)$ with $\kappa=0.01$ for (a) $t=10 \pi$, (b) $t=10 \pi+\pi / 4$, (c) $t=10 \pi+\pi / 2$, (d) $t=10 \pi+3 \pi / 4$, and (e) $t=11 \pi$ over one period of the flow with a source term $S=\cos x$.

$$
\frac{\partial C}{\partial t}+\frac{\partial F_{d}}{\partial A}=-\frac{\partial F_{S}}{\partial A}
$$

where $F_{d}$ is the diffusive flux and $F_{S}$ is the source flux. These are defined as 

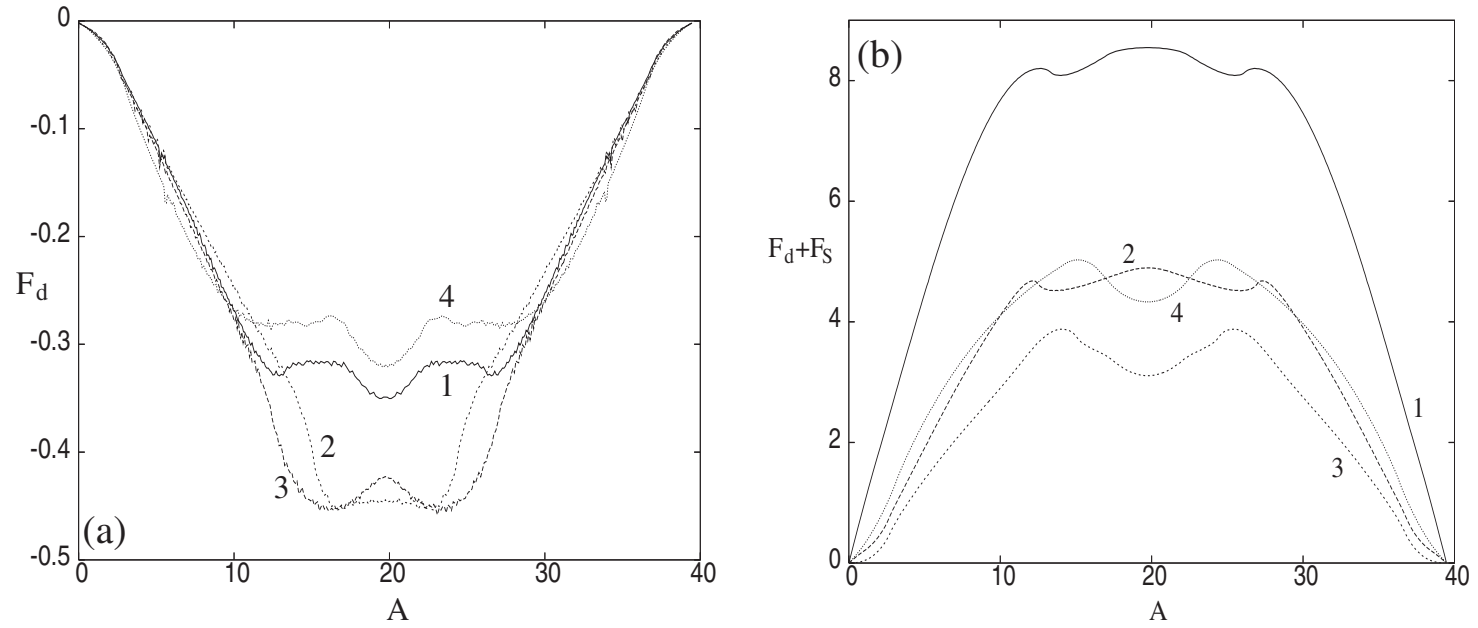

FIG. 11. Plot of the fluxes (a) $F_{d}(A)$ and (b) $F_{d}(A)+F_{S}(A)$ for the scalar field in Fig. 10 with lines $1-4$ representing times $t=10 \pi, 10 \pi+\pi / 4,10 \pi+\pi / 2$, and $10 \pi+3 \pi / 4$, respectively.

$$
\begin{aligned}
& F_{d}=-\kappa \frac{\left\langle\left|\nabla^{2} c\right|\right\rangle}{\partial C / \partial A}, \\
& F_{S}=-\int_{C \leq c(A, t)} S d A
\end{aligned}
$$

(see Ref. 1), and $F=-F_{d}$ from Eq. (9). In Fig. 11 we plot both (a) $F_{d}(A)$ and (b) $F_{d}(A)+F_{S}(A)$ for the scalar field depicted in Fig. 10 with the times $t=10 \pi, 10 \pi+\pi / 4$, $10 \pi+\pi / 2$, and $10 \pi+3 \pi / 4$ denoted by lines $1-4$, respectively. The diffusive flux in Fig. 11(a) contains some fine scale structure, but this is purely numerical. The diffusive flux has large gradients between $A=0$ and 10 and between $A=30$ and 40 for each time value, but between $A=10$ and 30 the diffusive flux increases in value for $t=10 \pi+\pi / 4$ and $t=10 \pi+\pi / 2$ and then decreases again as the peaks in $K_{\text {eff }}(A)$ reach their largest values, as in Fig. 10(d). This diffusive flux, however, is not solely driving the scalar distribution because we have the source term too. If we include the effect of this source term, then we have the flux which is plotted in
Fig. 11(b) and from this figure it is easier to describe how the effective diffusions in Fig. 10 govern the scalar evolution. In Fig. 11(b) the turning points of the flux appear to coincide with the peak values of $K_{\text {eff }}$, i.e., the saddle points in the scalar field. At $t=10 \pi$ (line 1) the flux gradient is mainly positive for $A<2 \pi^{2}$ and negative for $A>2 \pi^{2}$, so the scalar is being diffused away from the $C=0 \quad\left(A=2 \pi^{2}\right)$ contour. However, by $t=10 \pi+\pi / 2$ (line 3 ) the flux gradient has now changed sign close to $A=2 \pi^{2}$ and the scalar is now diffusing toward the $C=0$ contour.

Figure 12 plots (a) $K_{\text {eff }}(A)$ and (b) $C(A)$ at $t=10 \pi$ (solid line) and $t=11 \pi$ (dashed line). Figure 12(a) shows that at the beginning and end of each flow period the effective diffusion is approximately the same, even though over this period the scalar and the scalar flux vary considerably, as shown in Figs. 10 and 11(b). The variation of scalar concentration with $A$ over one period of the flow is given in Fig. 12(b). During the period this curve becomes modified as the scalar is rearranged, but after each period we see that the lines have a very similar appearance. For $13 \leqq A \leqq 27$ the two results are
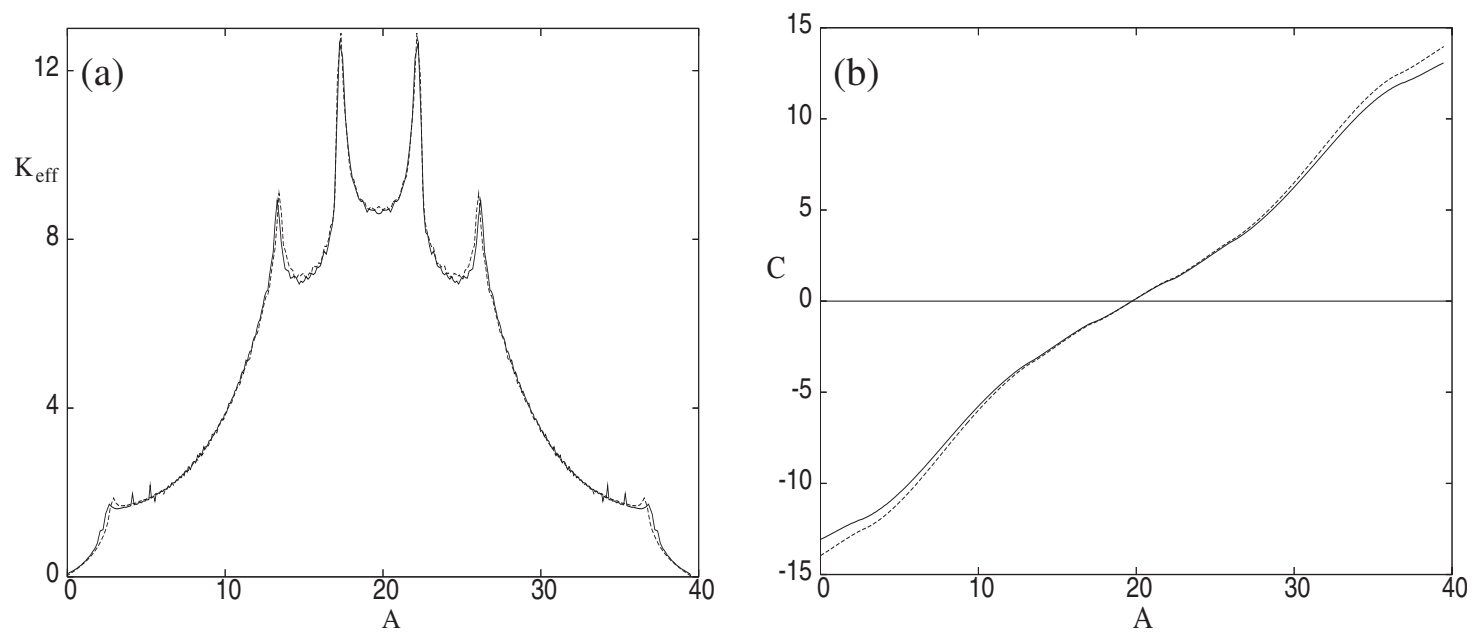

FIG. 12. Plot of (a) $K_{\text {eff }}(A)$ and (b) $C(A)$ for the scalar field in Fig. 10 at $t=10 \pi$ (solid line) and $t=11 \pi$ (dashed line) showing that the scalar distribution is now approximately periodic. 

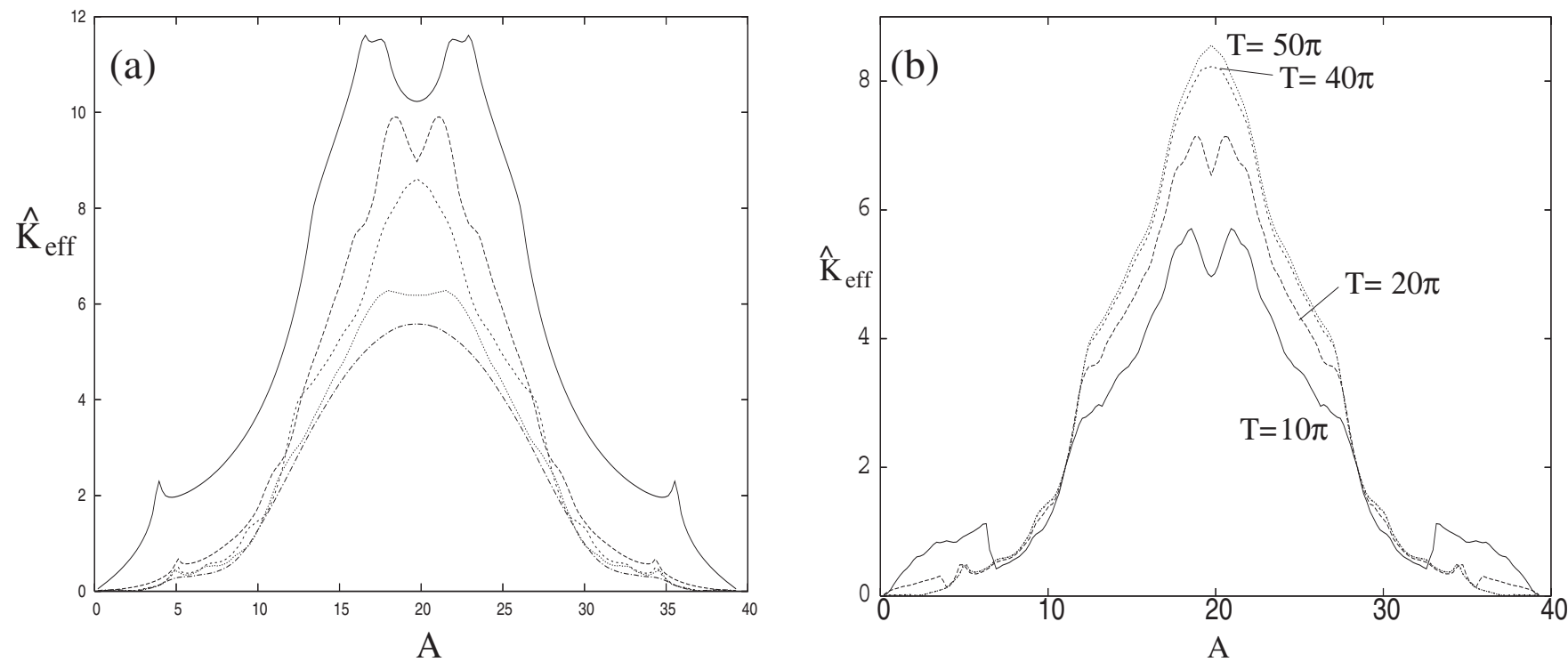

FIG. 13. Plot of (a) $\hat{K}_{\text {eff }}(A)$ for $\kappa=10^{-2}, 10^{-3}, 10^{-4}, 10^{-5}$, and $10^{-6}$ from top to bottom and (b) $\hat{K}_{\text {eff }}(A)$ for $\kappa=10^{-4}$ with $T=10 \pi, 20 \pi, 40 \pi$, and $50 \pi$ for a scalar field with a source term.

indistinguishable, showing that the injected scalar from the source term is mixed in this region, a region which is approximately the same as the region where the diffusion varies greatly over one period, see Fig. 11(a). For values of $A$ outside this region in Fig. 12(b) the value of the scalar increases in magnitude as the scalar builds up in island regions of low effective diffusion.

Although Fig. 10 shows how the effective diffusion varies over one period of the flow, it is difficult to interpret these figures into one average effective diffusion plot. To overcome this we define

$$
\hat{K}_{\text {eff }}=\overline{K_{\text {eff }} \frac{\partial C}{\partial A}} / \overline{\frac{\partial C}{\partial A}}
$$

to be the time-averaged effective diffusion where the bar signifies an average over one period of the flow. In Fig. 13(a) we plot $\hat{K}_{\text {eff }}(A)$ for various values of $\kappa$ ranging from $\kappa=10^{-2}$ (top line) to $\kappa=10^{-6}$ (bottom line). Each of these results was time averaged over the period from $T=50 \pi$ to $T+\pi$. By this time $K_{\text {eff }}$ has settled down into an approximate periodic state, and hence the time-averaged effective diffusion has appeared to settle down as shown in Fig. 13(b). This figure shows $\hat{K}_{\text {eff }}(A)$ for $\kappa=10^{-4}$ time averaged over one period starting at $10 \pi, 20 \pi, 40 \pi$, and $50 \pi$, and the difference between the $T=40 \pi$ and the $T=50 \pi$ results is small, showing that $K_{\text {eff }}$ probably reaches a steady state. For each value of $\kappa$ in Fig. 13(a) the largest effective diffusion values occur close to $A=2 \pi^{2}(C=0)$ and as $\kappa$ is reduced the two maximum peaks in $\hat{K}_{\text {eff }}(A)$ move toward this value. For $\kappa=10^{-4}$ these two peaks have become a single maximum in $\hat{K}_{\text {eff. }}$ As $\kappa$ is reduced it takes longer for the scalar in the islands to diffuse, and so $K_{\text {eff }}$ takes longer to settle down into a periodic state. The maximum value of $\hat{K}_{\text {eff }}$ reduces steadily with $\kappa$ and the difference between successive $\kappa$ values becomes small by the time $\kappa=10^{-6}$. This suggests that $\hat{K}_{\text {eff }}(A)$ and hence
$K_{\text {eff }}(A)$ may approach an inviscid limit; however, the results in Fig. 13(a) may not have settled down to a steady state, and Fig. 13(b) shows an extremely slow drift that needs to be examined on an $O\left(\kappa^{-1}\right)$ time scale. The investigation of this is beyond the scope of this paper and is left for future study with the use of simpler flows and mappings.

\section{CONCLUSIONS AND DISCUSSION}

In this paper we have explored the effective diffusion diagnostic for both idealized, given scalar fields and for advection of a passive scalar in a simple, periodic chaotic flow. We studied a two-dimensional scalar field which contains a saddle point and found that along scalar contours which pass through such saddle points the effective diffusion has a logarithmic singularity. This result was confirmed by twodimensional incompressible time-periodic simulations of the advection-diffusion equation. The numerical parameters were optimized to remove most of the numerical oscillations. These oscillations could be removed further by using a more accurate method for calculating $A(C)$ from Eq. (2) than the box counting technique chosen. However, the box counting method was sufficiently fast and accurate for the purpose of this study. We note that the peak in the effective diffusion that arises from saddle points can be considered a geometrical artifact: it is the fact that $\partial A / \partial C$ diverges at such points that makes $K_{\text {eff }}$ diverge. The actual flux at such contours remains finite.

By introducing a source of scalar in our numerical simulations we found that the scalar field exhibits an approximate periodic behavior with the period of the flow, and that in regions of high effective diffusion, the scalar concentration from the source term is well mixed. By considering a time average of these simulations we observe that as $\kappa$ is reduced, the effective diffusion still peaks around scalar concentration contours which occur in the chaotic bands of the scalar field. This study shows that the time-averaged effective diffusion 
appears to settle down to a steady state for large times, at least for moderate $\kappa$. The time-averaging process also removes the singular spikes from $K_{\text {eff }}$ and makes $\hat{K}_{\text {eff }}$ nonsingular; thus it is highly beneficial to time average $K_{\text {eff }}$ for flows of this type.

However, for small $\kappa$, the scalar distribution only converges on very long time scales, beyond our simulations, because of the presence of islands. The resulting $\hat{K}_{\text {eff }}$ initially converges rapidly in the chaotic region and then shows a slow drift with time. The time-averaged results presented in this study suggest the existence of an inviscid limit for $\hat{K}_{\text {eff. }}$. However, to confirm this Eq. (1) needs to be integrated to times of $O\left(\kappa^{-1}\right)$ to allow the scalar to reach a steady periodic state, or perhaps smaller values of $\kappa$ may need to be considered. Both of these are beyond our simulations and are left for future study.

\section{ACKNOWLEDGMENTS}

This work was supported by the EPSRC on Grant No. EP/D032202/1. A.D.G. is also grateful for the tenure of a Leverhulme Research Fellowship during this research. The authors would also like to thank the referees for their comments which have improved the paper.

\section{APPENDIX A: ASYMPTOTICS FOR A TWO-DIMENSIONAL SCALAR FIELD WITH A SADDLE POINT}

Here we consider the two-dimensional scalar field (7) with $|C| \ll 1$. Hence we are considering a concentration contour close to the saddle points at $(0,0)$ and $(-\pi,-\pi)$. We assume $0<C \ll 1$; however, a similar expression also holds for $-1 \ll C<0$ by symmetry. The region $A / 4$ given by Eq. (2) for this contour $C$ is given in Fig. 14. The value of $A$ for this value of $C$ can be split into three parts,

$$
\begin{aligned}
A(C) & =4\left(\int_{0}^{\pi-\rho} y d x+\int_{\pi-\rho}^{\rho} y d x+\int_{\rho}^{\pi} \pi d x\right) \\
& =A_{1}(C)+A_{2}(C)+A_{3}(C)
\end{aligned}
$$

where $y=\cos ^{-1}(\cos x-2 C)$ from Eq. (7) and $\rho=\cos ^{-1}(2 C-1)$. Using the fact that $C \ll 1$, we can approxi-

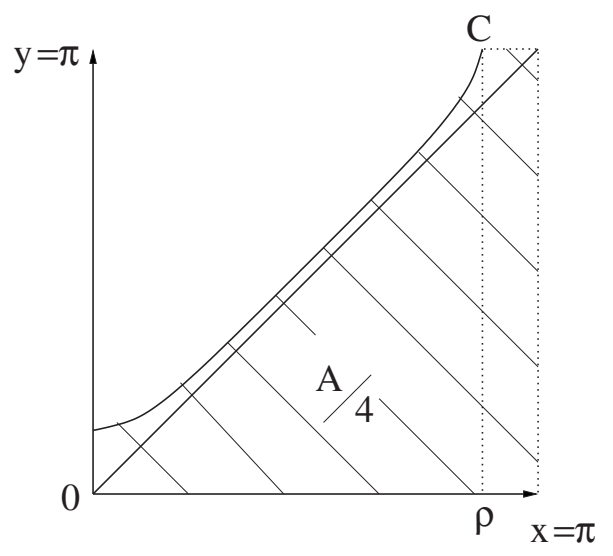

FIG. 14. Plot of the region $A / 4$ for scalar field (7) with the contour $0<C \ll 1$. mate these integrals and calculate the small $C$ form of $A(C)$. Clearly $A_{3}(C)$ can be evaluated explicitly as

$$
\begin{aligned}
A_{3}(C) & =4 \pi^{2}-4 \pi \cos ^{-1}(2 C-1) \\
& =8 \pi \sqrt{C}+\frac{4}{3} \pi C^{3 / 2}+O\left(C^{5 / 2}\right)
\end{aligned}
$$

for small $C$.

To evaluate the area $A_{2}(C)$ we expand the integrand $y=\cos ^{-1}(\cos x-2 C)$ for small $C$ to give

$$
\begin{aligned}
\cos ^{-1}(\cos x-2 C)= & x+\frac{2}{\left(1-\cos ^{2} x\right)^{1 / 2}} C \\
& -\frac{2 \cos x}{\left(1-\cos ^{2} x\right)^{1 / 2}} C^{2}+O\left(C^{3}\right)
\end{aligned}
$$

and integrate this with respect to $x$, which gives

$$
\begin{aligned}
A_{2}(C)= & 4\left[\frac{x}{2}\right]_{\pi-\rho}^{\rho} \\
& -8 C\left[\frac{\left(1-\cos ^{2} x\right)^{1 / 2} \tanh ^{-1}(\cos x)}{\sin x}\right]_{\pi-\rho}^{\rho} \\
& +8 C^{2}\left[\frac{1}{2 \sin x\left(1-\cos ^{2} x\right)^{1 / 2}}\right]_{\pi-\rho}^{\rho}+O\left(C^{3}\right) .
\end{aligned}
$$

Hence we find

$$
\begin{aligned}
A_{2}(C)= & -8 C \ln C+2 \pi^{2}-8 \pi \sqrt{C}-\frac{4}{3} \pi C^{3 / 2}-8 C^{2} \\
& +O\left(C^{5 / 2}\right) .
\end{aligned}
$$

A systematic approach in evaluating $A_{1}(C)$ would be to evaluate the integrand for small $x$, integrate this expression with respect to $x$, and finally expand the solution for small $C$. However, using this method we find that the leading order terms in the small $C$ expansion of $A_{1}(C)$ rely greatly on many terms of the small $x$ expansion of the integrand due to the nonuniformity of the series. Thus we approximate the leading order term of this integral by using the full numerical solution. We assume $A_{1}(C)=A_{11} C+o(C)$, and we calculate $A_{11}$ simply as

$$
A_{11}=\lim _{C \rightarrow 0} \frac{A-2 \pi^{2}+8 C \ln C}{C} \simeq 19.07 .
$$

Thus the first three terms in the small $C$ expansion for $A$ are $A(C)=A_{1}+A_{2}+A_{3}=-8 C \ln C+2 \pi^{2}+19.07 C+O\left(C^{2}\right)$, and hence

$$
\frac{\partial A}{\partial C}=-8 \ln C+11.07+O(C) .
$$

This shows that the effective diffusion has a logarithmic singularity at $C=0$ unless the integral $F(C)$ has a stronger singularity. Although the numerical solution in Fig. 2 shows that this is not the case, we calculate the small $C$ expansion for $F(C)$ for completeness; this process is outlined below.

As in the above analysis, we split the integral $F$ as 


$$
\begin{aligned}
F(C) & =2 \int_{0}^{\rho} I(x, C) d x \\
& =2 \int_{0}^{\pi-\rho} I(x, C) d x+2 \int_{\pi-\rho}^{\rho} I(x, C) d x=F_{1}+F_{2},
\end{aligned}
$$

where

$$
\begin{aligned}
I(x, C)= & \frac{\sin ^{2} x}{\left(1-\cos ^{2} x+4 C \cos x-4 C^{2}\right)^{1 / 2}} \\
& +\left(1-\cos ^{2} x+4 C \cos x-4 C^{2}\right)^{1 / 2} .
\end{aligned}
$$

To calculate $F_{2}(C)$ we expand the integrand for small $C$ and integrate, which gives

$$
F_{2}(C)=4 C^{2} \ln C+8-14 C+O\left(C^{2}\right) .
$$

This is an interesting result because the form of $F(C)$ in Fig. 2 is that of an even function; hence we do not expect to have any terms of $O(C)$ in our expansion. We show below that $F_{1}(C)$ is of $O\left(C^{2}\right)$, so at this point we neglect this $O(C)$ term as calculations not presented here show that it will be removed when we include more terms in the asymptotic expansion of the integrand.

We again use the numerical solution to evaluate $F_{1}(C)$ by assuming $F_{1}(C)=F_{11} C^{2}+o\left(C^{2}\right)$, and thus

$$
F_{11}=\lim _{C \rightarrow 0} \frac{F-8-4 C^{2} \ln C}{C^{2}} \simeq-15.1 \text {. }
$$

Therefore

$$
F(C)=F_{1}+F_{2}=4 C^{2} \ln C+8-15.1 C^{2}+O\left(C^{3}\right),
$$

which when combined with $\partial A / \partial C$, as in Eq. (4), gives

$$
K_{\text {eff }}(C)=-64 \ln C+88.56+O(C \ln C) \text {. }
$$

\section{APPENDIX B: EXACT SOLUTION OF $K_{\text {eff }}$ FOR A THREE-DIMENSIONAL SCALAR FIELD WITH A SEPARATRIX}

In this appendix we show that $K_{\text {eff }}$ is nonsingular at three-dimensional hyperbolic points. We consider a cylinder of a scalar tracer, with height 2 and radius 1 . Inside the cylinder are two cones which meet at the origin as depicted in Fig. 15. Inside the cones the scalar is positive and outside it is negative; hence the origin is a separatrix. The scalar distribution can be given analytically as

$$
c(r, \theta, z)=z^{2}-r^{2},
$$

where $(r, \theta, z)$ are the usual cylindrical polar coordinates. The volume of scalar corresponding to the concentration contour $C$ is given via Eq. (2) as

$$
V(C)=\int_{r=0}^{1} \int_{\theta=0}^{2 \pi} \int_{z=-1}^{1} H\left(C-z^{2}+r^{2}\right) r d r d \theta d z
$$

This quantity can be calculated numerically, but by the same argument as in Appendix A this integral and the corresponding three-dimensional version of $\int_{c \leq C(A, t)}|\nabla c|^{2} d A$ can be calculated exactly for this scalar field. Thus for this particular

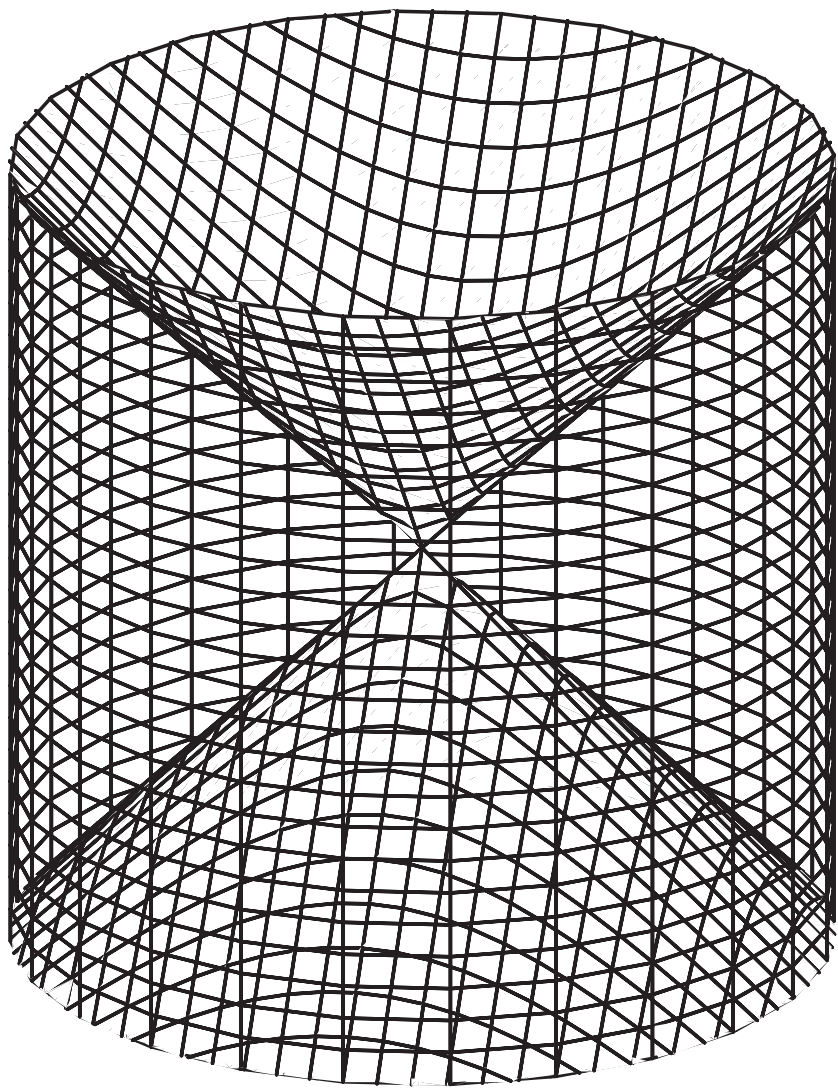

FIG. 15. A plot of the three-dimensional scalar field given in Eq. (B1). The inner cones represent the surface given by $C=0$, and inside these cones the scalar is positive and outside it is negative.

scalar field we have a closed form for $K_{\text {eff }}(C)$. The triple integral (B2) can be simplified to

$$
\begin{aligned}
V(C) & =\int_{0}^{2 \pi} \int_{0}^{1} z r d r d \theta \\
& =4 \pi \int_{0}^{(1-C)^{1 / 2}}\left(C+r^{2}\right)^{1 / 2} r d r+4 \pi \int_{(1-C)^{1 / 2}}^{1} r d r,
\end{aligned}
$$

which can be integrated to give

$$
V(C)=\frac{2 \pi}{3}\left(2+3 C-2 C^{3 / 2}\right) .
$$

When $C<0$ then the volume integral changes to

$$
\begin{aligned}
V(C) & =4 \pi \int_{(-C)^{1 / 2}}^{1} z r d r=4 \pi \int_{(-C)^{1 / 2}}^{1}\left(C+r^{2}\right)^{1 / 2} r d r \\
& =\frac{4}{3} \pi(1+C)^{3 / 2} .
\end{aligned}
$$

Thus

$$
V(C)= \begin{cases}\frac{4}{3} \pi(1+C)^{3 / 2}, & C<0, \\ \frac{2 \pi}{3}\left(2+3 C-2 C^{3 / 2}\right), & C>0 .\end{cases}
$$

For the evaluation of $F(C)$ we can convert the surface integral into an integral over $r$ and $\theta$. We again have to consider 

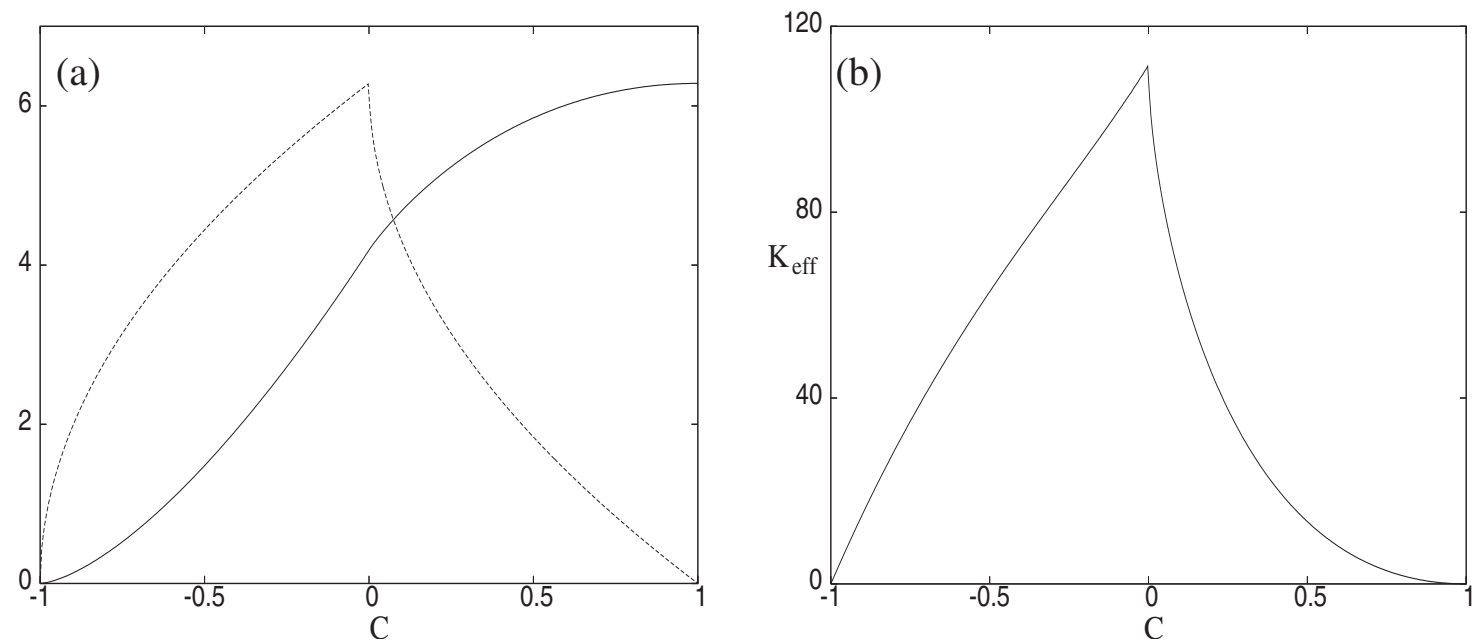

FIG. 16. Plot of (a) $V(C)$ (solid line) and $\partial V / \partial C$ (dashed line) and (b) $K_{\text {eff }}(C)$ for three-dimensional scalar field (B1).

the cases $C>0$ and $C<0$ separately, but we can get two exact solutions for this scalar field. When $C>0$ we have

$$
\begin{aligned}
F(C)= & 2 \int_{0}^{(1-C)^{1 / 2}} \int_{0}^{2 \pi}|\nabla c|\left[1+\left(\frac{d z}{d r}\right)\right]^{1 / 2} r d r d \theta \\
= & 8 \pi \int_{0}^{(1-C)^{1 / 2}}\left[\frac{\left(2 r^{2}+C\right)^{3}}{r^{2}+C}\right]^{1 / 2} r d r \\
= & \frac{\pi}{\sqrt{2}}\left\{2 \sqrt{2}(2-C)^{3 / 2}-3 \sqrt{2} C(2-C)^{1 / 2}+\sqrt{2} C^{2}\right. \\
& \left.+3 C^{2} \ln \left[\frac{\sqrt{2}+(2-C)^{1 / 2}}{(1+\sqrt{2}) C^{1 / 2}}\right]\right\} .
\end{aligned}
$$

For the case when $C<0$ then the only change to this calcu- lation is the limits of integration; thus the integral for $F$ becomes

$$
\begin{aligned}
F(C)= & 8 \pi \int_{(-C)^{1 / 2}}^{1}\left[\frac{\left(2 r^{2}+C\right)^{3}}{r^{2}+C}\right]^{1 / 2} r d r \\
= & \frac{\pi}{\sqrt{2}}\left\{2 \sqrt{2}(2+C)^{3 / 2}(1+C)^{1 / 2}\right. \\
& -3 \sqrt{2} C(2+C)^{1 / 2}(1+C)^{1 / 2} \\
& \left.+3 C^{2} \ln \left[\frac{(2+C)^{1 / 2}+\sqrt{2}(1+C)^{1 / 2}}{(-C)^{1 / 2}}\right]\right\} .
\end{aligned}
$$

Thus the effective diffusion for this three-dimensional problem is given as

$$
K_{\text {eff }}(C)= \begin{cases}\sqrt{2} \pi^{2}(1+C)^{1 / 2}\left\{2 \sqrt{2}(2+C)^{3 / 2}(1+C)^{1 / 2}-3 \sqrt{2} C(2+C)^{1 / 2}(1+C)^{1 / 2}+3 C^{2} \ln \left[\frac{(2+C)^{1 / 2}+\sqrt{2}(1+C)^{1 / 2}}{(-C)^{1 / 2}}\right]\right\}, & C<0, \\ \sqrt{2} \pi^{2}\left(1-C^{1 / 2}\left\{2 \sqrt{2}(2-C)^{3 / 2}-3 \sqrt{2} C(2-C)^{1 / 2}+\sqrt{2} C^{2}+3 C^{2} \ln \left[\frac{\sqrt{2}+(2-C)^{1 / 2}}{(1+\sqrt{2}) C^{1 / 2}}\right]\right\},\right. & C>0 .\end{cases}
$$

Figure 16 plots (a) $V(C)$ (solid line) and $\partial V / \partial C$ (dashed line) and (b) $K_{\text {eff }}(C)$ for the three-dimensional scalar field given by Eq. (B1). We note that in panel (a) both $V(C)$ and $\partial V / \partial C$ are continuous functions, but $V(C)$ has a discontinuity in its second derivative at $C=0$. Thus $\partial V / \partial C$ has no singularity at $C=0$ unlike in the two-dimensional case. Also as $F(C)$ has no singularities then $K_{\text {eff }}(C)$ in Fig. 16(b) has no singularity at $C=0$ either. Although there is no singularity in $K_{\text {eff }}$ at $C=0$, the maximum effective diffusion still occurs at this point, and so we may expect to see peaks in threedimensional effective diffusion plots, which will be important in the mixing of scalar tracers as for the twodimensional case in Sec. III, but this investigation is left as future study.

${ }^{1} \mathrm{~N}$. Nakamura, "Two-dimensional mixing, edge formation and permeability diagnosed in area coordinate," J. Atmos. Sci. 53, 1524 (1996).

${ }^{2}$ K. B. Winters and E. A. D'Asaro, "Diascalar flux and the rate of fluid mixing,” J. Fluid Mech. 317, 179 (1996). 
${ }^{3}$ P. Haynes and E. Shuckburgh, "Effective diffusion as a diagnostic of atmospheric transport. 1. Stratosphere," J. Geophys. Res. 105, 22777, DOI: 10.1029/2000JD900093 (2000).

${ }^{4} \mathrm{P}$. Haynes and E. Shuckburgh, "Effective diffusion as a diagnostic of atmospheric transport. 2. Troposphere and lower stratosphere," J. Geophys. Res. 105, 22795, DOI: 10.1029/2000JD900092 (2000).

${ }^{5}$ E. Shuckburgh and P. Haynes, "Diagnosing transport and mixing using a tracer-based coordinate system," Phys. Fluids 15, 3342 (2003)

${ }^{6}$ P. G. Drazin, Nonlinear Systems (Cambridge University Press, Cambridge, 1992).

${ }^{7}$ S. Wiggins, Introduction to Applied Nonlinear Dynamical Systems and Chaos (Springer, New York, 1990).

${ }^{8}$ A. J. Lichtenberg and M. A. Lieberman, Regular and Chaotic Dynamics, 2nd ed. (Springer, New York, 1992).

${ }^{9}$ R. T. Pierrehumbert and H. Yang, "Global chaotic mixing on isentropic surfaces," J. Atmos. Sci. 50, 2462 (1993).

${ }^{10}$ H. E. Deese, L. J. Pratt, and K. R. Helfrich, "A laboratory model of exchange and mixing between western boundary layers and subbasin recirculation gyres," J. Phys. Oceanogr. 32, 1870 (2002).

${ }^{11}$ M. R. Turner and A. D. Gilbert, "Linear and nonlinear decay of cat's eyes in two-dimensional vortices, and the link to Landau poles," J. Fluid Mech. 593, 255 (2007).

${ }^{12}$ N. Butchart and E. E. Remsberg, "Area of the stratospheric polar vortex as a diagnostic for tracer transport on an isentropic surface," J. Atmos. Sci. 43, 1319 (1986).

${ }^{13}$ N. F. Otani, "A fast kinematic dynamo in two-dimensional time-dependent flows," J. Fluid Mech. 253, 327 (1993).

${ }^{14}$ S. Childress and A. D. Gilbert, Stretch, Twist, Fold: The Fast Dynamo (Springer, Berlin, 1995).

${ }^{15}$ M. V. Berry and N. L. Balazs, "Evolution of semiclassical quantum states in phase space," J. Phys. A 12, 625 (1979).

${ }^{16}$ S. Ghosh, A. Leonard, and S. Wiggins, "Diffusion of a passive scalar from a no-slip boundary into a two-dimensional chaotic advection field," J. Fluid Mech. 372, 119 (1998). 